\title{
Multi-sensor data collection and fusion using autoencoders in condition evaluation of concrete bridge decks
}

\author{
Sepehr Pashoutani ${ }^{1}$, Jinying Zhu ${ }^{1 *}$ (D), Chungwook Sim¹', Kwanghee Won², Brian A. Mazzeo ${ }^{3}$ \\ and W. Spencer Guthrie ${ }^{4}$
}

\begin{abstract}
This paper presents a multi-sensor data collection and data fusion procedure for nondestructive evaluation/testing (NDE/NDT) of a concrete bridge deck. Three NDE technologies, vertical electrical impedance (VEI), ground-penetrating $\operatorname{radar}(\mathrm{GPR})$, and high-definition imaging (HDI) for surface crack detection, were deployed on the bridge deck. A neural network autoencoder was trained to quantify the relationship between VEI and GPR results using the data collected at common positions. This relationship was then used for fusion of VEI and GPR data to increase the reliability and spatial resolution of the NDE measurements and to generate a data-fused condition map that showed novel characteristics. Threshold values for VEI and GPR tests were obtained and used to determine the color scale in the fused map. Surface cracks identified from HDI show reasonable agreement with the deterioration areas on the data-fused condition map. Chloride concentration measurements on sound and deteriorated areas of the deck were consistent with the NDE results.
\end{abstract}

Keywords: Nondestructive testing, Data fusion, Ground-penetrating radar, Vertical electrical impedance, Autoencoder, Crack, Concrete, Bridge deck

\section{Introduction}

Concrete bridge decks are subject to deterioration caused by reinforcement corrosion, freeze-thaw cycling, construction deficiencies, traffic loads, and other factors. Nondestructive evaluation/testing (NDE/NDT) methods can provide quantitative assessment of both the extent and severity of such bridge deck deterioration, beyond that achieved by visual inspection [1]. With improvements in instruments and technologies, modern NDE tests have increased data acquisition speed and enhanced data quality. Although no single NDE method is yet able to fully characterize deterioration in reinforced concrete structures, using multiple NDE technologies in a synergistic manner may provide complementary information

\footnotetext{
*Correspondence: jyzhu@unl.edu

'Department of Civil and Environmental Engineering, University of

Nebraska-Lincoln, Omaha NE, 68182 USA

Full list of author information is available at the end of the article
}

and increase the reliability and accuracy of bridge deck condition assessments. The increasing availability and diversity of NDE methods provide new opportunities and challenges in data analysis and decision-making through aggregation of data from multiple sensing modalities.

Although inconsistency in multi-sensor NDE data can be a challenge when dealing with data collection in the field, data fusion, which involves aggregating complementary information, has been shown to be a viable solution [2-5]. Data fusion can be described as systematic integration of data from multiple sources to obtain more information than would be possible using a single source [6]. Data fusion techniques have been studied in multisensor NDE of bridge decks to quantify mechanical properties of materials [7], identify defects [8], and visualize multi-modal data [2].

In this research, three complementary NDE methodologies were used to evaluate a concrete bridge deck with a

\section{Springer Open}

(c) The Author(s). 2021 Open Access This article is licensed under a Creative Commons Attribution 4.0 International License, which permits use, sharing, adaptation, distribution and reproduction in any medium or format, as long as you give appropriate credit to the original author(s) and the source, provide a link to the Creative Commons licence, and indicate if changes were made. The images or other third party material in this article are included in the article's Creative Commons licence, unless indicated otherwise in a credit line to the material. If material is not included in the article's Creative Commons licence and your intended use is not permitted by statutory regulation or exceeds the permitted use, you will need to obtain permission directly from the copyright holder. To view a copy of this licence, visit http://creativecommons.org/licenses/by/4.0/. 
concrete overlay. The methodologies were vertical electrical impedance (VEI), ground-penetrating radar (GPR), and high-definition imaging (HDI) [9] for detecting surface cracks. VEI is a newly developed NDE system [10-12] that measures the electrical impedance of concrete from the deck surface to the nearest rebar mat at low frequencies $(\approx 100 \mathrm{~Hz})$. VEI is particularly sensitive to surface cracks that can provide a direct pathway for chloride ions to penetrate the concrete and accelerate corrosion of the reinforcement. GPR has been widely used for condition assessment of bridge decks by measuring rebar reflection amplitudes over the entire bridge deck. This technique also permits estimation of relative permittivity and electrical conductivity of concrete cover in the $\mathrm{GHz}$ frequency range.

Data fusion across NDE techniques can quantitatively increase bridge condition assessment resolution and accuracy beyond any single technique alone. The techniques in this work were selected because they can all be efficiently performed over relatively large areas. Because both VEI and GPR measure electrical properties of cover concrete, but at different frequencies and with different spatial resolution because of electrode configuration differences, their results are expected to show high correlation and provide complementary information. These similarities and differences make VEI and GPR ideal candidates for data fusion. This study demonstrates fusion of VEI and GPR data, enables quantitative determination of the nonlinear relationship between those two test results, and improves spatial resolution. The resulting data-fused map includes features that are not as visible in the individual VEI and GPR maps. As an additional technique, the vehicle-mounted HDI system allows fast collection of bridge deck surface images. Locations of surface cracks identified from the stitched images can be compared with VEI and GPR measurements to improve data interpretation, or HDI can be used as a stand-alone NDE method for quick screening evaluations of bridge decks. In this study, features identified from the fused VEI and GPR data are validated by the HDI images.

This paper briefly reviews the basic principles of VEI, GPR, and HDI techniques and then describes their application to field testing of a reinforced concrete bridge deck with a concrete overlay. Basic machine learning principles are then outlined. VEI and GPR results are presented separately in the form of condition maps, which are compared to chloride concentrations measured at two locations, and plotted on an image of the bridge deck. HDI-identified surface cracks are plotted on a stitched image of the bridge deck. Data fusion procedures for combining the information obtained from VEI and GPR tests are then presented. After an autoencoder-based data fusion technique was used to estimate the relationship between VEI magnitude and GPR amplitude, the transformed VEI and GPR data were mapped to common coordinates to generate a fused condition map that agrees well with the identified surface cracks.

\section{Methods for bridge deck evaluation Vertical electrical impedance}

VEI is an NDE technique used to quantitatively estimate the protection offered to steel reinforcement against chloride ion ingress [10-17]. A VEI measurement in a scanning configuration with a large-area electrode is shown schematically in Fig. 1. An alternating-current voltage potential $(100-1000 \mathrm{~Hz})$ is applied to the deck, and electrical current emanating from the center electrode of a guarded probe is measured. The applied voltage and measured current are used to estimate the impedance of the concrete cover from the bridge deck surface to the reinforcing steel. Impedance testing generally involves measurement of the in-phase (resistive) and out-of-phase (reactance) current that is measured when an alternating potential is applied to a material. Because an alternating potential is used, the frequency at which interrogation is performed is important [11].

VEI is a suitable method for characterizing cover protection because the electrical resistivity of concrete is largely a function of the properties of the concrete matrix and the pore water. A concrete matrix with high porosity characterized by high interconnectivity and low tortuosity allows for the passage of high amounts of electrical current and would have low resistivity compared to a concrete with low porosity. Regarding the pore water, high ion concentrations and high temperatures allow for the passage of high amounts of electrical current through the concrete due to the high abundance and mobility of current carriers. Because higher values of porosity, moisture content, chloride concentration, and temperature are all consistently correlated with higher corrosion rates, resistivity values can consequently be useful for identifying areas of deteriorating protection of the reinforcing steel. Low VEI indicates locations where the deck is susceptible to chloride ingress, rebar corrosion, and delaminations, or subsurface cracking, in the deck.

\section{Ground-Penetrating radar}

GPR is a widely used NDE method for localizing rebars and evaluating reinforced concrete condition. Figure 2 shows a typical GPR B-scan image recorded over a concrete bridge deck. Hyperbolic-shaped patterns in the image indicate reflections from rebars in the deck. The GPR antenna contains a transmitter module to emit electromagnetic pulses and a receiver to capture echoes from subsurface interfaces. The amplitude of the signals reflected from rebars depends on the electrical properties of the concrete through which the EM wave travels. Moisture and free charge carriers such as chloride ions 


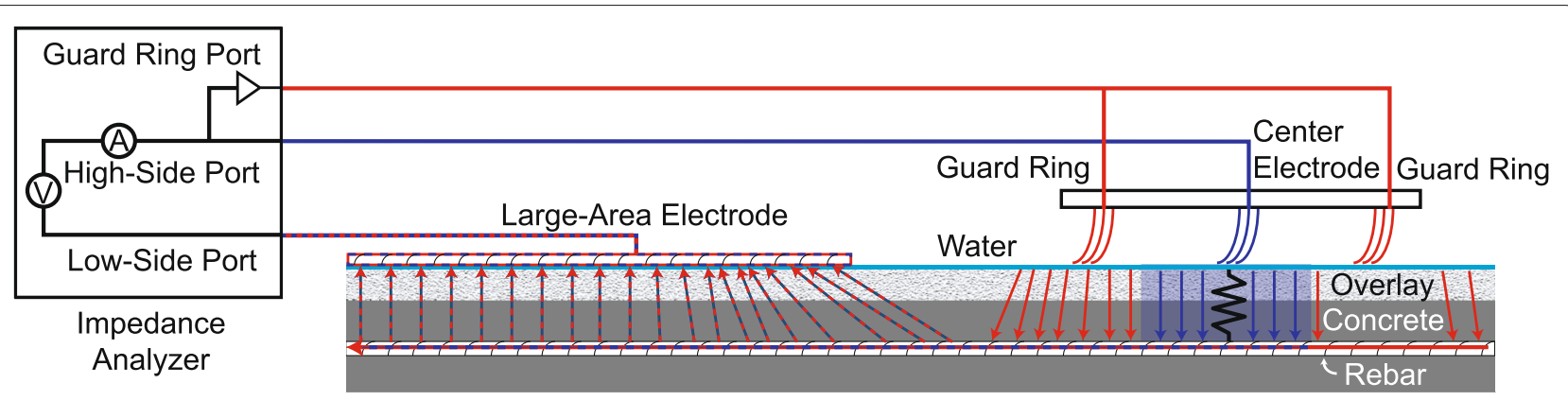

Fig. 1 Schematic of VEI measurement system consisting of an impedance analyzer connected to a probe and a large-area electrode

increase the attenuation of the signal. Therefore, analysis of the reflection amplitude is commonly used to identify regions with a high probability of corrosion-induced deterioration.

In this work, GPR data were analyzed by applying zero time offset and migration and then normalized by surface direct-coupling amplitude with depth correction. Zero time offset and migration of rebar reflections are used to detect rebars and estimate the electromagnetic wave velocity profile. The rebar reflection amplitudes are further normalized by the surface direct-coupling amplitude over each rebar to remove the effect of surface conditions and then corrected based on the rebar depths that were calculated from the wave velocity and travel time. The surface normalization and real depth correction improve the accuracy of GPR data analysis. Details are described in recent publications by the authors $[18,19]$.

\section{High-Definition imaging}

In this study, HDI included data acquisition, localization, and detection of deficiencies which in this study were surface cracks on the bridge deck. For data acquisition, four machine-vision cameras attached to a vehicle were used to collect high-resolution images from the bridge deck as shown in Fig. 3.

Although a global positioning system (GPS) and an odometer sensor can be used for localization, GPS signals are not always available or stable in some urban areas, and GPS does not provide pose information. In addition, odometer sensor data can provide relative position along the direction of scanning but not in the perpendicular direction. Because GPS and odometer sensor data may not work for every case, the system [9] employed in this research used non-contact optical sensors to complete the localization task.

Among the four cameras, two were used to simplify the localization process with a wide field-of-view (angle) that helped localize image frames in both the longitudinal (direction of traffic) and transverse directions. The two large field-of-view cameras captured the full width of the bridge deck and helped compensate for the relative pose changes during the stereo reconstruction of 3D point clouds.

Two other cameras having a deeper focal length were used to capture detailed high-resolution images to support the detection task. These cameras were able to pro-



Fig. 2 GPR B-scan image that is captured with a 1.5-GHz antenna over a concrete bridge deck 


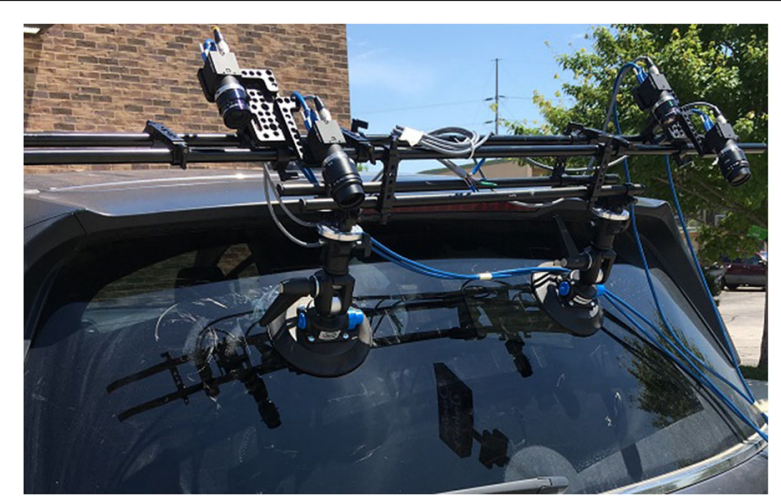

Fig. 3 High-resolution images collected from machine-vision cameras mounted on a vehicle

vide twice the resolution of the other pair of cameras, which allowed detection of cracks with a width of approximately $0.25 \mathrm{~mm}$; this width is considered to be about half of the average crack width typically observed on bridge decks [20].

\section{Chloride concentration testing}

Along with NDE tests of the bridge, chloride concentration testing was also performed at two locations on the bridge deck. Chloride concentration measurement is a destructive evaluation technique that involves drilling into the bridge to a particular depth, pulverizing the concrete into powder, carefully collecting the pulverized powder, and subsequently titrating the powder samples in the laboratory to estimate the chloride concentration in the concrete at particular depths. Chloride concentration testing can provide ground-truth comparisons for other NDE measurements.

\section{Autoencoders}

Neural networks (NN) are a major class of machine learning algorithms in which the computer uses optimization techniques to adjust network parameters to create computational relationships between observed features and their target values. NNs have been used to model complex phenomena and solve regression problems and have been applied to NDE of materials [21, 22]. In supervised learning, every set of parameters is labeled with its corresponding response. Unsupervised learning, on the other hand, is based on finding the relationship between observations based on similarities or dissimilarities between observations without labeling.

Autoencoders are unsupervised NNs that are trained to replicate the input data. Applications of autoencoders include dimensionality reduction [23, 24], noise removal $[25,26]$, and nonlinear principal component analysis [27]. Figure 4 shows the architecture of a prototypical type of deep autoencoder with multiple hidden layers. The NN consists of two main parts: Encoder and Decoder. The Encoder transforms the input data into the Code layer, which has fewer neurons than the Input layer. The Decoder part is trained to reconstruct the original inputs from the Code layer outputs. Reducing the input dimensions in the Code layer prevents the NN parameters from reaching an identity function. Through several optimization iterations, the Encoder part learns to preserve and transform important features, and the Decoder part learns to invert these features to substantially represent the original data. This architecture can allow the network to reduce the input dimension for better interpretation with no major loss of information. Assuming that input noise does not have patterned features that can be learned by the NN, the NN output will have less noise.

\section{Field test results}

A three-span, two-lane, steel-girder bridge was selected for the NDE field demonstration. The reinforced concrete bridge deck has a total length of $55 \mathrm{~m}$, a width of 12.1

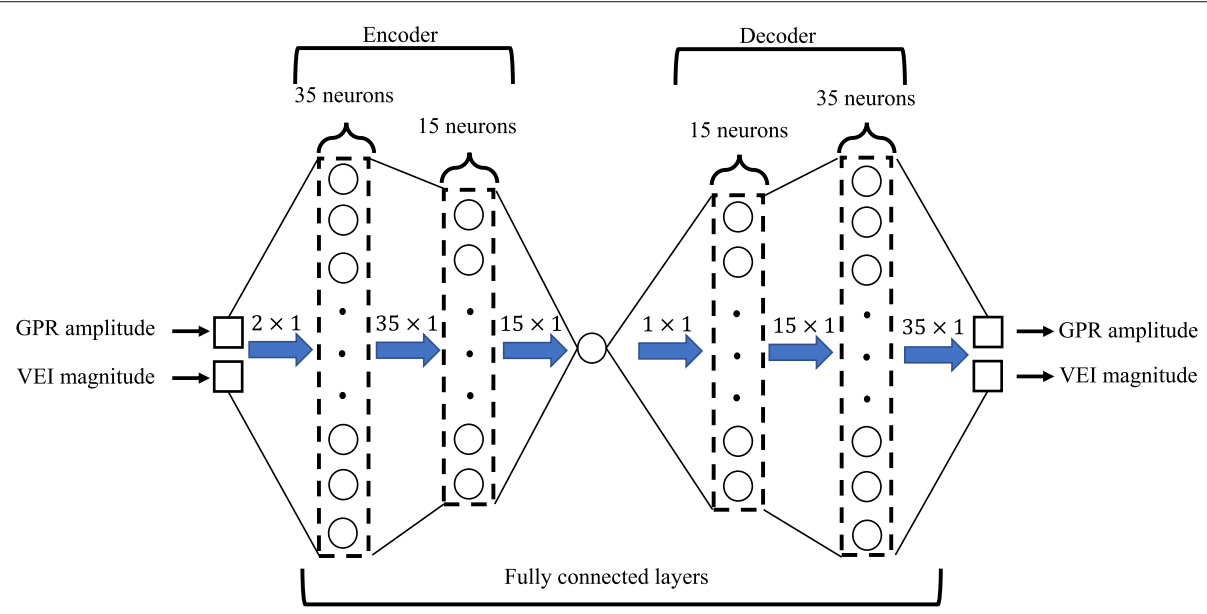

Fig. 4 Autoencoder diagram with fully connected layers 
$\mathrm{m}$, and a $5 \mathrm{~cm}$-thick concrete overlay. A satellite image of the bridge is shown in Fig. 5. Due to constraints associated with traffic control provided for testing, only one lane and the adjacent shoulder were inspected, as highlighted in Fig. 5.

The unique modes of data acquisition determined the order of data collection. Because the vehicle-mounted HDI system could scan the bridge deck at a normal traffic speed, HDI was performed first. Since GPR results could be affected by the water applied for VEI, GPR measurements were obtained second. VEI was the last nondestructive test performed on the bridge.

The locations of VEI measurements were recorded by using a combination of GPS, light detection and ranging (LIDAR), and imaging. The locations of GPR measurements were recorded by a real-time kinematic (RTK) GPS system. After all of the NDE data were collected on the bridge deck, VEI maps produced on-site were used to guide collection of concrete powder samples taken for chloride concentration testing at two locations and at two depths on the bridge deck, one in an area of higher VEI magnitude and one in an area of lower VEI magnitude.

\section{Vertical electrical impedance results}

The VEI system used in this study consisted of a vehicletowed apparatus with six parallel channels spaced approximately $0.6 \mathrm{~m}$ apart [11]. Two scanning passes were recorded along the bridge deck in the direction of traffic. Figure 6 shows the map of measured VEI impedance. The map shows consistent transverse lines characterized by low impedance across the bridge deck. It also has a longitudinal line characterized by low impedance approximately $3 \mathrm{~m}$ from the parapet wall. This line corresponded to a construction joint present on the bridge shoulder line. Another main feature of the impedance map is the occurrence of multiple large areas of low impedance near both ends of the bridge. Although these areas exhibited no visually apparent defects, a chain-drag test indicated that these locations were delaminated. Lastly, areas along the shoulders of the deck in which debris had accumulated also correlated spatially with some of the low-impedance areas on the map; these areas may have been more susceptible to salt accumulation during winter.

\section{Ground-Penetrating radar results}

A GSSI SIR-4000 GPR system with a $1.5 \mathrm{GHz}$ antenna was used in this study. Ten GPR scans were recorded along the bridge deck in the direction of traffic, with a line spacing of $0.6 \mathrm{~m}$. The longitudinal resolution of GPR scanning was fixed at $3 \mathrm{~mm}$, and the recorded signal time was $15 \mathrm{~ns}$. The positions of the GPR scans were recorded using a rover RTK GPS device mounted on a survey cart. GPR signals were processed using the previously described algorithm developed by the authors [18]. After migration, the longitudinal resolution of the GPR scans was equal to the rebar spacing, which is $12.5 \mathrm{~cm}$ in the traffic lane and $25 \mathrm{~cm}$ in the shoulder. Figure 7 shows the GPR amplitude map of the bridge deck. Low amplitude means high attenuation of the GPR signals, which may indicate deterioration of concrete cover and/or corrosion of rebars. Scattered high-attenuation regions are observed from the left joint to $15 \mathrm{~m}$ on the bridge deck. From $40 \mathrm{~m}$ to the right joint, four major high-attenuation areas were detected. These high-attenuation regions were consistent with low-impedance areas indicated on the VEI map.

Although the VEI and GPR maps have different background colors, they highlight similar defect regions. The data range and color scale used in each figure are based on either the maximum and minimum values in the data set or on experience. With the 'jet' colormap used in this study, warm color regions are interpreted as deterioration, suggesting that the VEI map indicates more deterioration than the GPR map. The difference between the VEI and GPR maps results from the different sensitivities inherent in the measurements and the different threshold values used in each map; determination of proper thresholds continues to be a challenging topic in many NDE methods. In this paper, the authors employ machine learning algorithms to determine unified values to relate VEI and GPR and use these values to prepare a fused-data map.

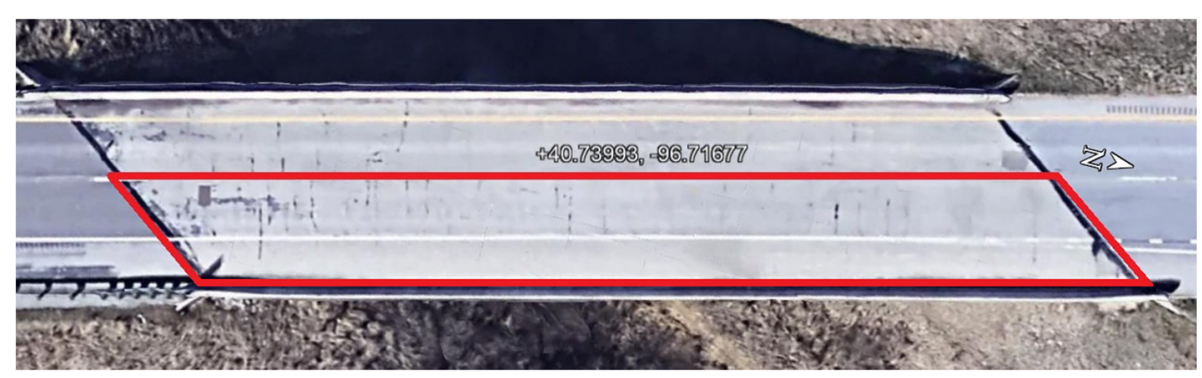

Fig. 5 Satellite image of bridge S077 05693R (map data @2018 Google) 


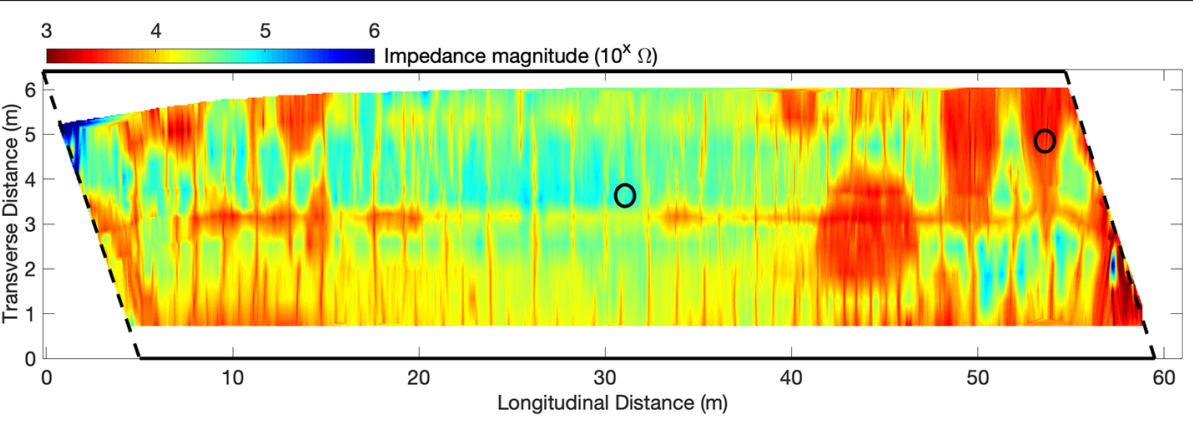

Fig. $6 \mathrm{VEI}$ map of the bridge deck (locations of chloride sampling are indicated by circles)

\section{High-Definition imaging results}

Compared to many other image-based crack-detection algorithms applied to a local region, this study focused on implementation of the detection method reported in [9] on a larger scale for the entire bridge deck. While most crack-detection methods rely only on detecting line segments and their intensities, this study created a hierarchy of crack pixels and crack segments to detect cracks using a circular histogram to capture not only the crack presence but also the crack orientation.

The detection of cracks was completed in two steps. First, crack pixels were detected by examining the local image patches centered at each pixel. For each pixel, a circular histogram from the image patches was built to check whether the pixel had a dominant orientation of low-intensity pixels or not. This information allows the orientation of the detected cracks to be determined. Second, from each detected crack pixel, crack segments were formed if the detected crack pixel exhibited the following features: 1) supporting cracks pixels were within a local window defined by a specific radius, 2) crack pixels had similar orientation, and 3) the relative direction of the crack pixels was consistent with the orientations of multiple crack pixels and the crack pixel of interest. A crack segment was formed when a minimum number of supporting pixels within a local window were observed for a crack pixel. A detailed description of crack identification and sample images are provided in [9].

Based on this hierarchy of crack pixels and crack segments, Fig. 8 shows the global map of all detected cracks on the entire bridge deck. Approximately 30 transverse cracks were detected for this $55 \mathrm{~m}$ bridge, indicating that the average spacing between these transverse cracks was approximately $1.8 \mathrm{~m}$. Considering rebar spacing and concrete cover, which are two parameters that are considered in crack control, the expected crack spacing was estimated to be $0.3 \mathrm{~m}$. Therefore, the detected transverse cracks were spaced more widely than expected. The difference may be at least partially attributable to the presence of the overlay, through which only a portion of the cracks in the deck may have reflected. The VEI data indicate more frequent and longer transverse deterioration segments across the deck, which suggest that VEI was sensitive to early-stage deterioration conditions that may eventually result in cracking observable by HDI.

\section{Chloride concentration results}

Two chloride concentration sampling locations, marked by circles in Fig. 6, were selected based on the VEI results obtained initially at the test site. On-site VEI map generation highlighted the rapid automated processing, localization, and mapping algorithms developed for VEI scanning

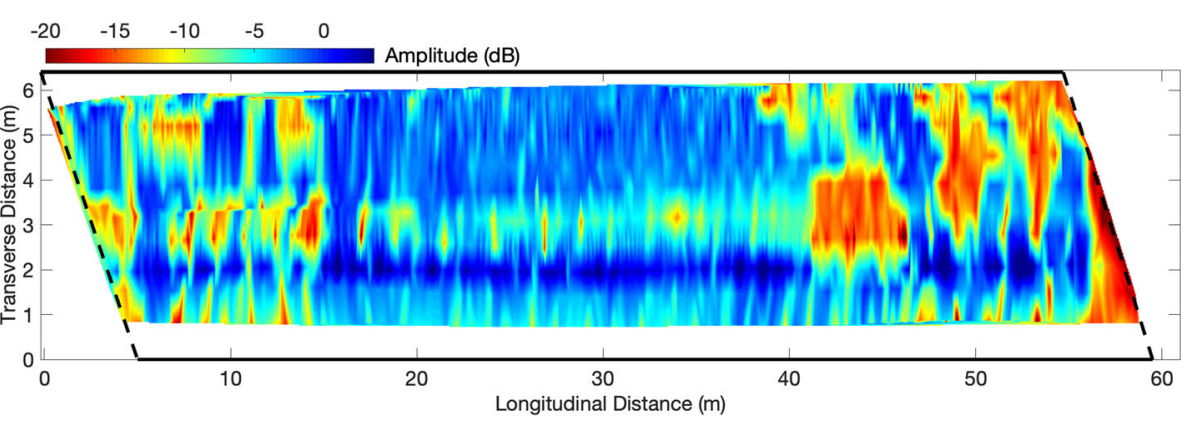

Fig. 7 GPR amplitude map of the bridge deck 




(a)

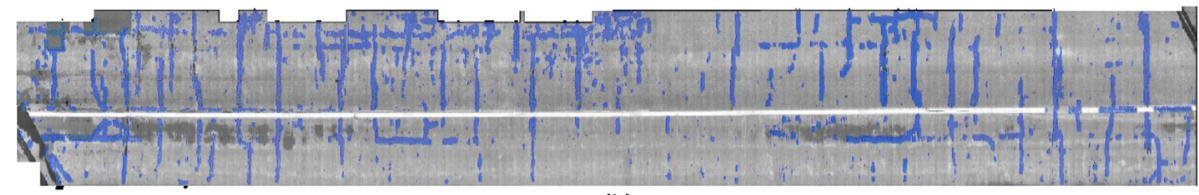

(b)

Fig. 8 (a) Stitched HDI image of the bridge deck and (b) identified surface cracks overlapped on the bridge deck image (the blue markers only give the crack locations and do not represent crack widths)

[11]. The low-VEI sampling location was positioned at a longitudinal distance of $54 \mathrm{~m}$ and a transverse distance of $4.7 \mathrm{~m}$, and the high-VEI sampling location was positioned at a longitudinal distance of $31 \mathrm{~m}$ and a transverse distance of $3.6 \mathrm{~m}$. The results of the chloride concentration measurements are shown in Table 1 . As expected, a high chloride concentration corresponded to a lower VEI and lower GPR amplitude, as shown in Fig. 7.

\section{Data fusion results and discussion Data fusion procedures}

In reinforced concrete structures, the corrosion rate of the reinforcement depends to a large degree on the moisture content and chloride concentration of the concrete [13]. Because VEI magnitude and GPR amplitude are both significantly influenced by moisture content and chloride concentration $[10,28]$, the results of VEI and GPR testing are expected to be strongly correlated. In the field testing, the scanning paths for the VEI and GPR testing were not exactly aligned, as shown in Fig. 9, and the corresponding data have different spatial resolutions. Data fusion of these two data sets would therefore be expected to provide improved spatial resolution and complementary information about the bridge deck condition. The data fusion analysis included two steps: (1) determine the statistical relationship between VEI and GPR measurements using NN analysis and (2) fuse transformed VEI and GPR data to the same reference framework.

\section{Data preparation}

In order to determine the statistical relationship between VEI and GPR measurements, VEI and GPR data collected from the same or nearby positions needed to be spatially associated. Because the VEI data have higher spatial resolution, VEI values within a $12.5 \mathrm{~cm}$ radius of rebar were averaged for each migrated GPR amplitude above a rebar. This GPR amplitude and the averaged VEI magnitude were then regarded as a data pair obtained from the same position. After all data points were extracted from overlapped positions, outliers were removed using a simple quantile-based flooring and capping (2.5\% and 97.5\%) method based on their histograms. Figure 10a shows the scatter plot of the GPR amplitudes and VEI impedance and histograms of each data set (3562 data points).

\section{Autoencoder training}

Before training, the logarithm of VEI magnitude and GPR amplitude $(\mathrm{dB})$ were linearly normalized (MinMax scaling) to the range of $[0,1]$, which corresponds to the unscaled VEI range of $10^{3.4} \sim 10^{4.9} \Omega(2.5-83 \mathrm{k} \Omega)$ and GPR range of $[-16.6,2.4] \mathrm{dB}$. After training, the normalized output data were scaled back to their original data ranges.

In Fig. 10a the calculated Pearson's correlation coefficient is 0.603 with a corresponding $p$-value $\approx 0$. Although this correlation is significant with respect to statistical hypothesis testing, it cannot explain the nonlinear

Table 1 Chloride concentration test results

\begin{tabular}{|c|c|c|c|c|c|}
\hline Long. Dist. (m) & Trans. Dist. (m) & Ave. Depth (mm) & Cl. Conc. * & $\operatorname{VEI}(\Omega)$ & GPR Amp. (dB) \\
\hline \multirow{2}{*}{31} & \multirow{2}{*}{3.6} & 12.5 & 4.0 & \multirow{2}{*}{79,000} & \multirow{2}{*}{-3.1} \\
\hline & & 40 & 1.1 & & \\
\hline \multirow{2}{*}{54} & \multirow{2}{*}{4.7} & 12.5 & 13.3 & \multirow{2}{*}{2,500} & \multirow{2}{*}{-13.8} \\
\hline & & 40 & 9.0 & & \\
\hline
\end{tabular}

*(lb Cl- $/ y d^{3}$ of Concrete) 




Fig. 9 Scanning paths of VEl and GPR tests recorded by GPS (the VEI scanning path were curved near the start and end points)

relationship between VEI and GPR. Therefore, an autoencoder was designed as a function to represent this relationship. With an autoencoder, a prior assumption regarding the intercorrelation of multi-sensor measurements within the data is not necessary. For the autoencoder, two neurons for the Input layer (VEI and GPR measurements) and one for the Code layer (combined features) were defined in Fig. 4. Multiple models with varying numbers of neurons in the hidden layers were trained, and, based on the average mean-square error (MSE) of the four-fold cross-validation sets (75\% for training and 25\% for testing), the model with the least average MSE was selected. In the optimal model, the Encoder part consisted of 35 and 15 neurons for the first and second hidden layers, respectively. The Decoder part is a mirror replica of the Encoder part with respect to the Code layer. The initial learning rate for training was set to 0.005 . The learning rate was updated through the training phase when the loss value did not improve for five iterations.

Figure 10b plots the Output layer values from the autoencoder after the data were scaled back to the original data ranges. Although Fig. 10b has the same number of data points as Fig. 10a, the transformed data shows much less scattering and gives a clear nonlinear relationship between the VEI and GPR data. The histograms on each axis give distributions of the inputs and outputs, which show some outliers were compressed.

\section{Threshold values}

The scatter plot in Fig. 10a shows a high concentration of data points in the VEI ranges of $10^{4} \sim 10^{4.7} \Omega(10-50 \mathrm{k} \Omega)$ and GPR amplitude range of $[-6,0.3] \mathrm{dB}$. The VEI condition map (Fig. 6) shows that the threshold impedance is around $10^{4} \Omega$, which corresponds to both the impedance


Fig. 10 Scatter plot of VEI vs. GPR data collected at the same locations: (a) original input data and (b) autoencoder output (unscaled), where the original data were normalized before input to the autoencoder model 
value for deterioration concern reported in other studies [11] and the lower bound of the data range with high concentration in Fig. 10a. According to the literature, the lower bound of the GPR data cluster with high concentration corresponds to a moderate corrosion threshold amplitude [29]. The lower bound value, $-6 \mathrm{~dB}$ in Fig. 10a, is also close to a threshold value of $-5.3 \mathrm{~dB}$ previously reported by the authors [18]. These values are also in agreement with the chloride concentration measurement results in Table 1. Therefore, in this study, the threshold values for VEI and GPR are chosen as $10 \mathrm{k} \Omega$ and $-6 \mathrm{~dB}$, respectively.

\section{Data fusion results}

With the equation shown in Fig. 10b, either VEI or GPR data can be transformed to combine these two data sets. Since the VEI data have better spatial resolution in the longitudinal direction, all GPR data were converted to VEI values, and then the two data sets were combined. If both VEI and GPR values were available at the same coordinates, the average value was used. A MATLAB function 'griddata' was used to interpolate scattered data points in the combined data set on a common coordinate frame.

Figure 11 shows the map generated from the fused VEI and GPR values on the bridge deck, for which the threshold value for VEI magnitude was set as $10 \mathrm{k} \Omega$ as previously explained. In 'jet' color maps, light green is usually used to represent a neutral value or a threshold value to differentiate between sound and deteriorating regions; therefore, a light green color was assigned to the threshold of $10 \mathrm{k} \Omega$ in Fig. 11. If the VEI data were instead converted to GPR values to generate a fused map, then the GPR threshold of -6 $\mathrm{dB}$ would be used. When the proper threshold values are used in the VEI, GPR, and fused maps, all maps will have a similar background color.

The fused data set contains integrated information from both the VEI and GPR measurements and has increased spatial resolution. Compared to the individual VEI and GPR maps shown in Figs. 6 and 7, the fused map not only contains features from each map, but it also reveals additional features that could not be clearly observed in the individual maps. For example, in the region of 20 $38 \mathrm{~m}$ in the longitudinal direction and above $5 \mathrm{~m}$ in the transverse direction, the fused map shows horizontally oriented deterioration areas that are not as visible in the VEI or GPR maps. These deterioration areas might correspond to longitudinally oriented cracks. The fused map also shows several other longitudinal deterioration areas below the shoulder line (about $2.5 \mathrm{~m}$ in the transverse direction) in the regions of 5-15 $\mathrm{m}$ and 40-60 $\mathrm{m}$ in the longitudinal direction. The fused data remain consistent with the chloride concentration measurements.

Surface cracks on the bridge deck provide pathways for chloride ions to penetrate into the concrete and cause rebar corrosion and concrete deterioration. Figure 12 overlaps the HDI-identified concrete surface cracks on the fused VEI/GPR map. Several general patterns are observed. First, in the regions of 0-15 m and 40-55 m in the longitudinal direction, there are many long transverse cracks, which match the deck deterioration areas indicated by the VEI and GPR data. Second, many deterioration areas are visible at $3 \mathrm{~m}$ in the transverse direction, which is along the longitudinal construction joint between the driving lane and the shoulder. This result indicates that the construction joint also provides a pathway for moisture and chloride ion penetration. Third, many longitudinal crack segments were identified by the HDI technique not only at $2.5 \mathrm{~m}$ but also above $5 \mathrm{~m}$ in the transverse direction. These cracks are in good agreement with the deterioration features on the fused map that are not as visible on the individual VEI and GPR maps in Figs. 6 and 7.

The fused image in Fig. 12 gives much more useful information than a single NDE technique could provide.

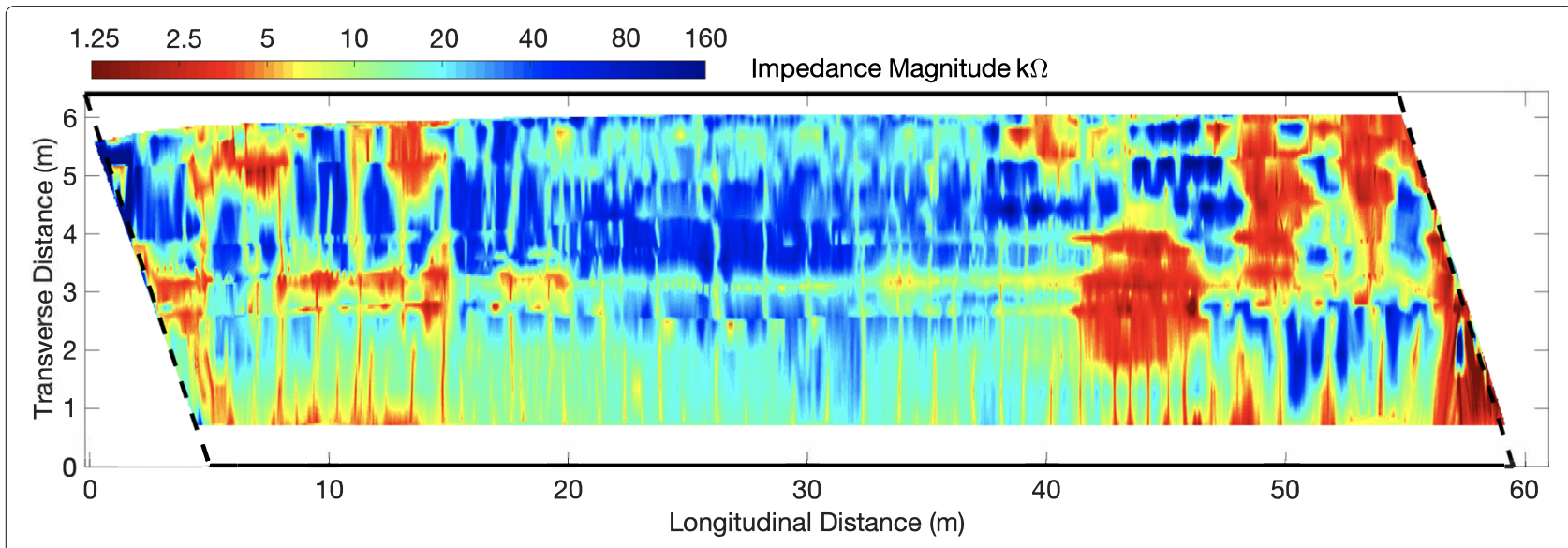

Fig. 11 Fused VEI and GPR map (GPR amplitude was converted to VEl magnitude using the equation obtained in the autoencoder analysis, with the threshold for VEI magnitude set at $10 \mathrm{k} \Omega$ ) 


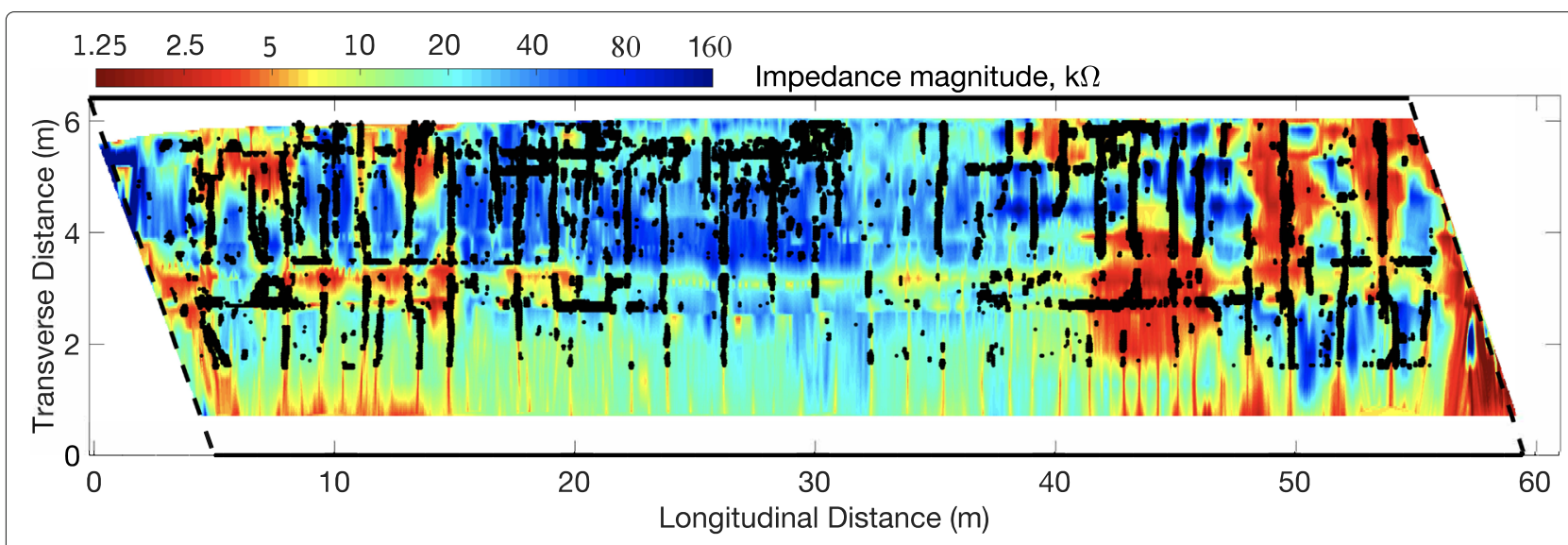

Fig. 12 Identified cracks from HDI images overlapped on the fused VEI/GPR map

Comparison of surface cracks with the fused VEI/GPR image provides a better understanding of the effects of surface cracks on concrete deterioration and rebar corrosion. Fusing independent VEI and GPR test data not only improves the spatial resolutions but it also reveals additional features that could be missed by individual VEI and GPR tests. Confirmation of these additional features by HDI-detected cracks further validates the effectiveness of the data-fusion algorithm. In future work, crack width information may be extracted and correlated with the fused map condition estimates.

\section{Conclusions}

This research presents a multi-sensor data collection and data fusion study based on three NDE methodologies, including VEI, GPR, and HDI. An autoencoder-based data fusion was implemented on the GPR and VEI results obtained from a concrete bridge deck to quantify their relationship, which was then used to combine both data sets and increase the spatial resolution and accuracy of the overall condition map. The fused data were then compared to the surface cracks obtained by HDI and the chloride concentration test results. The main findings from this work are summarized as follows:

1 Both the VEI and GPR methods measure electrical properties of reinforced concrete. VEI is a relatively new NDE technique for measuring the electrical impedance of concrete, and GPR amplitudes are sensitive to changes in the electrical conductivity of concrete. Therefore, a strong correlation was expected between these two measurements. This hypothesis is verified in this study using experimental data collected on a concrete bridge deck with a concrete overlay for which VEI and GPR testing provided similar results for bridge deck evaluation.

2 A neural network model can be used to quantify the relationship between VEI and GPR data by using VEI and GPR data collected from the same positions. With the established nonlinear relationship from the autoencoder, either VEI or GPR data can be transformed to each other and combined in the same coordinates to form a fused NDE map.

3 The density scatter plot shows a VEI threshold of about $10 \mathrm{k} \Omega$ and a GPR threshold value around -6 $\mathrm{dB}$, which are consistent with previous findings by the authors $[11,18]$. Use of proper threshold values in all NDE maps is important for achieving consistent visualization results.

4. The fused map contains features from each data set and reveals additional features that are not as visible in the individual NDE maps. Longitudinally-oriented cracks and deterioration that are parallel with the NDE scan paths may be missed by one NDE test. However, data fusion increases the possibility of detection of these types of defects.

5 Visible cracks on the concrete surface can be direct pathways for water and chloride ion to reach the steel reinforcement as suggested by the results of chloride concentration testing. High correlation also exists between the presence of major cracks and deterioration estimates from VEI and GPR testing. This finding highlights the importance of sealing cracks in concrete bridge deck maintenance. VEI and GPR are also suggested as useful tools to predict eventual crack formation, specifically when data fusion is applied to their combined data sets.

\section{Abbreviations}

NDE/NDT: Nondestructive Evaluation/Testing; VEl: Vertical Impedance; GPR: Ground Penetration RADAR; HDI: High Definition Imaging; GPS: Global Positioning System; RTK: Real-time Kinematic; LIDAR: Light Detection and Ranging; NN: Neural Network; MSE: Mean-square Error

\section{Acknowledgements}

The authors acknowledge the Nebraska Department of Transportation (NDOT) for financial support and NDOT engineers Mark Traynowicz, Fouad Jaber, Jason Volz, Kent Miller, and their colleagues for providing the bridge information and assisting in the field testing. BYU students Jared Baxter and Lorin Hendricks assisted in field testing. 


\section{Authors' contributions}

SP developed the GPR analysis software, acquired GPR and GPS data, analyzed the data, composed figures, and prepared the original draft. JZ acquired the funding, supervised the research project, designed the methodology, and substantially contributed to writing the manuscript. CS acquired the high-definition images, contributed to writing the manuscript, and reviewed the manuscript. KW acquired and analyzed the high-definition images. BM and SG developed the VEI system, acquired VEl and chloride concentration data, and contributed to writing the manuscript. All authors read and approved the final manuscript.

\section{Funding}

This research was funded by the Nebraska Department of Transportation. BM and SG also received support from the National Cooperative Highway Research Program IDEA Program.

\section{Availability of data and materials}

The data sets used and/or analyzed during the current study are available from the corresponding author on reasonable request.

\section{Declarations}

\section{Competing interests}

The authors declare that they have no competing interests.

\section{Author details}

${ }^{1}$ Department of Civil and Environmental Engineering, University of Nebraska-Lincoln, Omaha NE, 68182 USA. ${ }^{2}$ Department of Electrical Engineering and Computer Science, South Dakota State University, Brookings, SD, USA. ${ }^{3}$ Department of Electrical and Computer Engineering, Brigham Young University, Provo, UT, USA. ${ }^{4}$ Department of Civil and Environmental Engineering, Brigham Young University, Provo, UT, USA.

\section{Received: 10 March 2021 Accepted: 6 June 2021}

Published online: 22 June 2021

\section{References}

1. Gucunski N, Imani A, Romero F, Nazarian S, Yuan D, Wiggenhauser H, Shokouhi P, Taffe A, Kutrubes D (2013) Nondestructive testing to identify concrete bridge deck deterioration. Transportation Research Board SHRP 2 Report S2-R06A-RR-1

2. Kohl C, Streicher D (2006) Results of reconstructed and fused NDT-data measured in the laboratory and on-site at bridges. Cem Concr Compos 28(4):402-413. https://doi.org/10.1016/j.cemconcomp.2006.02.005

3. Kee S-H, Oh T, Popovics JS, Arndt RW, Zhu J (2012) Nondestructive bridge deck testing with air-coupled impact-echo and infrared thermography. J Bridg Eng 17(6):928-939. https://doi.org/10.1061/(ASCE)BE.1943-5592. 0000350

4. Oh T, Kee S-H, Arndt RW, Popovics JS, Zhu J (2013) Comparison of NDT methods for assessment of a concrete bridge deck. J Eng Mech 139(3):305-314. https://doi.org/10.1061/(ASCE)EM.1943-7889.0000441

5. Islam MM, Kim J-M (2019) Vision-based autonomous crack detection of concrete structures using a fully convolutional encoder-decoder network. Sensors 19:4251. https://doi.org/10.3390/s19194251

6. Hall DL, Llinas J (1997) An introduction to multisensor data fusion. Proc IEEE 85(1):6-23. https://doi.org/10.1109/5.554205

7. Sbartaï Z-M, Breysse D, Larget M, Balayssac J-P (2012) Combining NDT techniques for improved evaluation of concrete properties. Cem Concr Compos 34(6):725-733. https://doi.org/10.1016/j.cemconcomp.2012.03. 005

8. Abu Dabous S, Yaghi S, Alkass S, Moselhi O (2017) Concrete bridge deck condition assessment using ir thermography and ground penetrating radar technologies. Autom Constr 81:340-354. https://doi.org/10.1016/j. autcon.2017.04.006

9. Won K, Sim C (2020) Automated transverse crack mapping system with optical sensors and big data analytics. Sensors 20(7):1838. https://doi. org/10.3390/s20071838

10. Guthrie WS, Baxter JS, Mazzeo BA (2018) Vertical electrical impedance testing of a concrete bridge deck using a rolling probe. NDT E Int 95:65-71. https://doi.org/10.1016/j.ndteint.2018.01.006
11. Mazzeo BA, Guthrie WS (2019) Vertical electrical impedance scanner for concrete bridge deck assessment without direct rebar attachment. Transportation Research Board, NCHRP-IDEA Program Project Final Report No. 202. http://www.trb.org/Main/Blurbs/179622.aspx

12. Baxter JS, Hendricks LJ, Guthrie WS, Mazzeo BA (2020) Instrumentation for multi-channel vertical electrical impedance scanning of concrete bridge decks. Eng Res Expr 2(3):035010

13. Bartholomew PD, Guthrie WS, Mazzeo BA (2012) Vertical impedance measurements on concrete bridge decks for assessing susceptibility of reinforcing steel to corrosion. Rev Sci Instrum 83(8):085104. https://doi. org/10.1063/1.4740479

14. Argyle HM (2014) Sensitivity of electrochemical impedance spectroscopy measurements to concrete bridge deck properties. Master's thesis, Brigham Young University-Provo. http://hdl.lib.byu.edu/1877/etd6875

15. Guthrie WS, Mazzeo BA (2015) Vertical impedance testing for assessing protection from chloride-based deicing salts provided by an asphalt overlay system on a concrete bridge deck. In: 16th International Conference on Cold Regions Engineering, Salt Lake City, Utah, July 19-22, 2015. pp 358-369. https://doi.org/10.1061/9780784479315.032

16. Baxter JS, Guthrie WS, Waters T, Barton JD, Mazzeo BA (2018) Vertical electrical impedance evaluation of asphalt overlays on concrete bridge decks. In: AIP Conference Proceedings Vol. 1949. p 030011. https://doi. org/10.1063/1.5031534

17. Barton J, Baxter J, Guthrie WS, Mazzeo BA (2019) Large-area electrode design for vertical electrical impedance scanning of concrete bridge decks. Rev Sci Instrum 90(2):025101. https://doi.org/10.1063/1.5058152

18. Pashoutani S, Zhu J (2020) Ground penetrating radar data processing for concrete bridge deck evaluation. J Bridg Eng 25(7):04020030. https://doi. org/10.1061/(ASCE)BE.1943-5592.0001566

19. Pashoutani S, Zhu J (2019) Condition assessment of concrete bridge deck with asphalt overlay. Nebraska Department of Transportation Final Report SPR-P1(17) M065. https://trid.trb.org/view/1687979

20. FroschFollow RJ, Blackman DT, Radabaugh RD (2003) Investigation of bridge deck cracking in various bridge superstructure systems. Indiana Department of Transportation Final Report FHWA/IN/JTRP-2002/25. https://doi.org/10.5703/1288284313257

21. Ebrahimkhanlou A, Dubuc B, Salamone S (2019) A generalizable deep learning framework for localizing and characterizing acoustic emission sources in riveted metallic panels. Mech Syst Signal Process 130:248-272. https://doi.org/10.1016/j.ymssp.2019.04.050

22. Hendricks LJ, Baxter JS, Chou Y, Thomas M, Boekweg E, Guthrie WS, Mazzeo BA (2020) High-speed acoustic impact-echo sounding of concrete bridge decks. J Nondestruct Eval 39(3):58

23. Zabalza J, Ren J, Zheng J, Zhao H, Qing C, Yang Z, Du P, Marshall S (2016) Novel segmented stacked autoencoder for effective dimensionality reduction and feature extraction in hyperspectral imaging. Neurocomputing 185:1-10. https://doi.org/10.1016/j.neucom.2015.11. 044

24. Pathirage CSN, Li J, Li L, Hao H, Liu W, Ni P (2018) Structural damage identification based on autoencoder neural networks and deep learning. Eng Struct 172:13-28. https://doi.org/10.1016/j.engstruct.2018.05.109

25. Gondara $L$ (2016) Medical image denoising using convolutional denoising autoencoders. In: 2016 IEEE 16th International Conference on Data Mining Workshops (ICDMW). pp 241-246. https://doi.org/10.1109/ ICDMW.2016.0041

26. Araki S, Hayashi T, Delcroix M, Fujimoto M, Takeda K, Nakatani T (2015) Exploring multi-channel features for denoising-autoencoder-based speech enhancement. In: 2015 IEEE International Conference on Acoustics, Speech and Signal Processing (ICASSP). pp 116-120. https:// doi.org/10.1109/ICASSP.2015.7177943

27. Licciardi GA, Khan MM, Chanussot J, Montanvert A, Condat L, Jutten C (2012) Fusion of hyperspectral and panchromatic images using multiresolution analysis and nonlinear PCA band reduction. EURASIP J Adv Signal Process, 207. https://doi.org/10.1186/1687-6180-2012-207

28. Lai W-L, Kind T, Stoppel M, Wiggenhauser H (2013) Measurement of accelerated steel corrosion in concrete using ground-penetrating radar and a modified half-cell potential method. J Infrastruct Syst 19(2):205-220. https://doi.org/10.1061/(ASCE)IS.1943-555X.0000083

29. Dinh K, Zayed T, Moufti S, Shami A, Jabri A, Abouhamad M, Dawood T (2015) Clustering-Based Threshold Model for Condition Assessment of Concrete Bridge Decks with Ground-Penetrating Radar. Transp Res Rec 2522(1):81-89. https://doi.org/10.3141/2522-08 


\section{Publisher's Note}

Springer Nature remains neutral with regard to jurisdictional claims in published maps and institutional affiliations.

Submit your manuscript to a SpringerOpen ${ }^{\circ}$ journal and benefit from:

- Convenient online submission

- Rigorous peer review

- Open access: articles freely available online

- High visibility within the field

- Retaining the copyright to your article

Submit your next manuscript at $>$ springeropen.com 